\title{
Therapeutic Effect of a Novel Human Parathyroid Hormone Analog Nasal Drops on Osteoporosis Rats
}

\author{
Jiao Feng ${ }^{a, b}$,Yan Zhu ${ }^{a}$,Shuang Gao ${ }^{a}$, Shuyan Ren ${ }^{a}$, and Rongyue Cao ${ }^{b, *}$ \\ ${ }^{a}$ Biological Engineering Department, Liaoning Economic Management Cadre Institute, \\ Shenyang, 110122, P. R. China \\ ${ }^{\mathrm{b}}$ Laboratory of Minigene Pharmacy, School of Life Science and Technology, China \\ Pharmaceutical University, NO. 24 Tongjia Xiang, Nanjing 210009, P. R. China \\ *Corresponding author: Rongyue Cao, Doctor, fengjiao720@163.com
}

\begin{abstract}
In this study, the therapeutic effect of a novel human parathyroid hormone analogs nasal drops on ovariectomized rats was evaluated. Thirty-two of 40 3-month-old female Sprague-Dawley rats were ovariectomized and 8 received sham operation. Thirteen weeks later, ovariectomized rats were randomly and averagely divided into 4 groups: low dose, middle dose and high dose group (31.25, 125, $500 \mu \mathrm{g} / \mathrm{rat}$ respectively) and vehiculum group (0.5 mL/rat). Each group was administered to the nasal drops. Another 8 sham-operated rats were treated in the same way as those of vehiculum group. All rats were administered daily. After 16 weeks of continuous administration, the osteogenic ability was compared between rats in each group. The results indicated that compared with vehiculum group, significant increments in the dry weights of femur, trabecular area and trabecular width were shown in the groups treated with middle dose and high dose hPTH'. The results showed dose dependence. It is concluded that hPTH' nasal drop is effective in stimulating bone formation and improving skeletal microarchitecture and potentially an effective therapeutical agent on osteoporosis.
\end{abstract}

Key words: human parathyroid hormone; nasal drops; osteoporosis; trabecula of bone

\section{Introduction}

As a new therapeutical agent for the treatment of osteoporosis via daily subcutaneous


more simple preparation and lower cost than human parathyroid hormone (1-34) [hPTH(1-34)] (F. Jiao et al. ${ }^{1}$ ). However, long-term and persistent injections resulted in pains of patients and thus decreased compliance. Therefore, an alternative approach to injection, such as nasal administration, is in great demand.

At present, the nasal drops of peptide and protein drugs have strong clinical advantages. It is a rapidly developed in recent years. The drug solution (including suspension) was directly injected into the nasal cavity without special administration device. Patients could complete nasal drug delivery with lower risk of a single overdose (E. Marttin et al. ${ }^{2}$, S. Salatin et al. ${ }^{3}$ ). It is supposed that hPTH' nasal drops should improve the compliance and reduce the pain of patients. In this study, the therapeutic effect of nasal drops of hPTH' on osteoporosis in OVX rats was studied. 


\section{Materials and methods}

\subsection{Materials and animals}

hPTH' was synthesized in the Laboratory of Minigene Pharmacy, China Pharmaceutical University, with purity $>96 \%$. Forty 10 -week old female Sprague-Dawley rats were purchased from Shanghai SIPPR-BK Lab animal Co., Ltd., China (licence no. SCXK20070005). All animals received humane care as described in the Principles of Laboratory Animal Care formulated by China Pharmaceutical University.

\subsection{Preparation of ' $P$ PTH' nasal drops}

The vehiculum of nasal drops was prepared by dissolving DM- $\beta-C D(40 \mathrm{mg} / \mathrm{mL})$, polyacrylic acid $(2.5 \mathrm{mg} / \mathrm{mL})$ and EDTA-2Na $(0.1 \mathrm{mg} / \mathrm{mL})$ into saline and adjusting solution $\mathrm{pH}$ to 6 . hPTH' was dissolve into the nasal drops excipients. The high dose hPTH' nasal drops (1 $\mathrm{mg} / \mathrm{mL})$, middle dose hPTH' nasal drops (250 g/mL), low dose hPTH' nasal drops $(62.5 \mathrm{~g} / \mathrm{mL})$ were prepared.

\subsection{Experimental protocol}

Animals received food and water on an ad libitum basis, and lighting lasted on a 12-h cycle. After two-week stabilization, 32 rats were ovariectomized (OVX) and eight were adopted all the other operations except OVX (group Sham). Thirteen weeks later, OVX rats were randomly assigned to 4 groups $(\mathrm{n}=8$ ): vehiculum group (group Vehiculum), low-dose groups (group hPTH'inL), middle-dose groups (group hPTH'inM), high-dose groups (group hPTH'inH). The four groups were nasally administrated with vehiculum, low dose hPTH' nasal drops, middle dose hPTH' nasal drops and high dose hPTH' nasal drops, respectively. Another eight rats (group Normal) without any operation and those of group Sham were treated in the same way as those of group OVX. All rats were nasally administrated on consecutive days for 16 weeks. Body weights were measured weekly and doses were adjusted accordingly.

\subsection{Bone histomorphology}

The samples of femoral bones were visualized by a S-3000N scanning electron microscope (SEM) (Japan) at accelerating voltage of $20 \mathrm{kV}$. The marrow space area and trabecular width were measured under the 70 and 300 SEM, respectively. In addition, all the results were analyzed by a morphological microimage analysis system (JD801, China).

\subsection{Statistical analysis}

All values are expressed as mean \pm S.D. . A one-way ANOVA was performed with SPSS 15.0 to detect statistical differences. Differences within the ANOVA were determined using a Tukey's post-hoc test. $P$ value of less than 0.05 was considered to be statistically significant.

\section{Results and Discussion}

\subsection{Dry weight of femur}

Compared with group Sham, the femoral weight of rats in group Vehiculum decreased 8.1\% $(P<0.01)$. Compared with group Vehiculum, the femoral weight of rats in group hPTH'inL, hPTH'inM and hPTH'inH were increased 2.8\% $(P>0.05), 12 \%(P<0.01)$ and $15.1 \%(P<$ 
0.001). The results showed dose dependence. The femur weights of rats in group hPTH'inH increased 8.2\% compared with the group Sham $(P<0.05)$.

\subsection{Bone histomorphology}

Bone quality is usually assessed by histomorphometry to prevent unintentional augmentation in fracture risk, even if BMD increase occurs. The $70 \times$ SEM and $300 \times$ SEM were used to visualize the sagittal plane of femoral bone. Fig. 1 and Fig.2 demonstrated that the marrow space area of groups treated with hPTH' nasal drops decreased, while trabecular widths increased. The mean marrow space area and trabecular width of each group are shown in Table 1. Compared with group Sham, both indices of hPTH' nasal drops-treated groups significantly changed $(P<0.01)$. With respect to marrow space area, the decreased percentages of hPTH' nasal drops-treated groups were 8.6\% $(P>0.05), 14.5 \%(P<0.05)$ and 26.2\% $(P<0.01)$ and showed dose dependence. In addition, with respect to trabecular widths, the increased percentages of hPTH' nasal drops-treated groups were 35.8\% $(P<0.01), 54.0 \%$ $(P<0.001)$ and $74.4 \%(P<0.001)$ and showed dose dependence. However, in comparison with group Sham, remarkable increments in trabecular width were shown only in all the hPTH' nasal drops-treated groups, while marked decrement in marrow space area was shown only in group hPTH'inM and hPTH'inH. It suggested that group hPTH'inL could maintain skeletal microarchitecture while group hPTH'inM and hPTH'inH were able to increase bone turnover and improve skeletal microarchitecture.

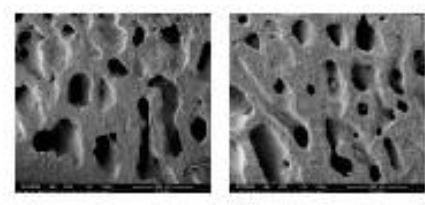

(A) Sham

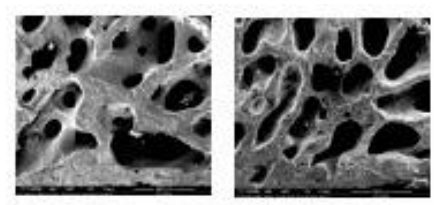

(B) Vehiculum

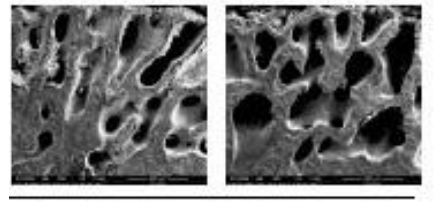

(C) hPTH'in L

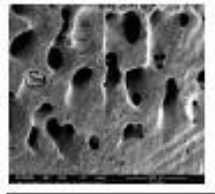

(D) $\mathrm{hPTH}$ 'in M

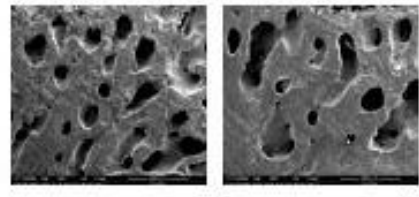

(E) $\mathrm{hPTH}$ 'inH

Fig. 1-The appearances of femur sagittal plane were visualized by 70× SEM (n=2). (A), (B) Shamoperated and OVX rats, treated with vehiculum, respectively; (D), (E), (F) OVX rats nasally treated with $\mathrm{hPTH}$ at $3.125,12.5$ and $50 \mu \mathrm{g} / \mathrm{rat}$, respectively.

\subsection{Bone formation markers}

The ALP activity in serum of each group is also shown in Table 1. Compared with group Sham, ALP activities of all administration groups increased significantly. The increased percentages of group Vehiculum and hPTH' nasal drops-treated groups were $47.5 \%(P<$ 0.05), 47.6\% $(P<0.05)$, $66.5 \%(P<0.05)$ and 97.8\% $(P<0.01)$, respectively.

\subsection{Discussion}

With the development of biotechnology, peptides are widely used in clinic. Because of the instability of peptides in vivo and vitro, its main clinical dosage is injection and freeze-dried powder. Experimental studies have indicated that the administration of hPTH' via daily 
subcutaneous injection effectively stimulated bone formation. However, long-term and persistant injections resulted in pains and thus decreased compliance. Therefore, its alternative approach to injection is in great demand. The nasal drug delivery has the advantages of convenience and good compliance. It is especially suitable for long-term treatment.

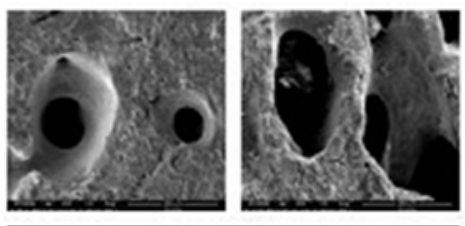

(A) Sham



(B) Vehiculum

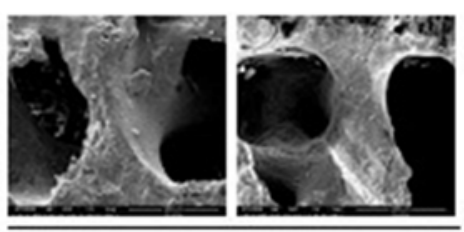

(C) hPTH'in L

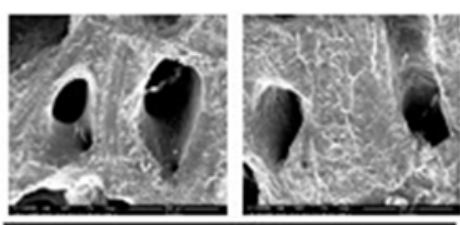

(D) $\mathrm{hPTH}$ 'inM

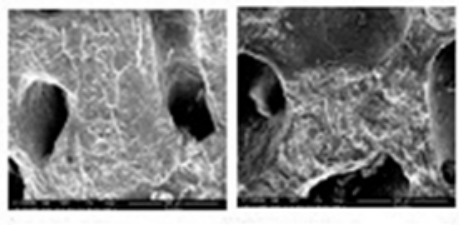

(E) hPTH'inH

Fig. 2- The appearances of femur sagittal plane were visualized by 300× SEM (n=2). (A), (B) Shamoperated and OVX rats, treated with vehiculum, respectively; (D), (E), (F) OVX rats nasally treated with hPTH' at 3.125, 12.5 and $50 \mu \mathrm{g} / \mathrm{rat}$, respectively.

Table 1 - The trabecular area and trabecular width of femur $(\mathrm{n}=8)$

\begin{tabular}{|c|c|c|c|}
\hline Group & $\begin{array}{c}\text { Trabecular area of femur } \\
\left(\mathrm{mm}^{2}\right)\end{array}$ & $\begin{array}{c}\text { Trabecular width of femur } \\
(\mu \mathrm{m})\end{array}$ & $\begin{array}{c}\text { Alkaline phosphatase activity } \\
(\mathrm{U} / \mathrm{L})\end{array}$ \\
\hline Sham & $1.957 \pm 0.050$ & $117.6 \pm 16.7$ & $147.8 \pm 37.6$ \\
\hline Vehiculum & $1.616 \pm 0.149$ & $80.4 \pm 3.9$ & $218.0 \pm 58.4^{\mathrm{b}}$ \\
\hline hPTH'inL & $1.755 \pm 0.143^{\text {a }}$ & $109.2 \pm 11.1^{\text {aa }}$ & $218.2 \pm 24.9^{\mathrm{b}}$ \\
\hline hPTH'inM & $1.850 \pm 0.046^{\text {aa }}$ & $123.8 \pm 15.2^{\text {aaa }}$ & $246.1 \pm 50.6^{\text {b }}$ \\
\hline hPTH'inH & $2.039 \pm 0.057^{\text {aaa }}$ & $140.2 \pm 12.5^{\text {aa, b }}$ & $292.3 \pm 61.8^{\text {bb }}$ \\
\hline
\end{tabular}

${ }^{\text {a }} P<0.05$ for the comparison with group Vehiculum. ${ }^{\text {aa }} P<0.01$ for the comparison with group Vehiculum. ${ }^{\text {aaa }} P$ $<0.001$ for the comparison with group Vehiculum. ${ }^{\mathrm{b}} P<0.05$ for the comparison with group Sham. ${ }^{\text {bb }} P<0.01$ for the comparison with group Sham.

The results showed that the treatment effect of $1000 \mu \mathrm{g}$ hPTH(1-34) nasal drops was the same as $20 \mu \mathrm{g}$ that of subcutaneous injection on osteoporosis. However, 500 and $250 \mu \mathrm{g} \mathrm{hPTH}(1-34)$ nasal drops are ineffective in the treatment of osteoporosis (T. Matsumoto et al. ${ }^{4}$ ). The conversion ratio between human dose and rat dose was 0.018:1 (X. Shuyun et al. ${ }^{5}$ ). Assuming that a person weighing $60 \mathrm{~kg}$ and a rat weighing $300 \mathrm{~g}$, the lowest effective dose of hPTH (134 ) in the treatment of rats osteoporosis should be $31.5 \mu \mathrm{g} / \mathrm{rat}$. It is higher than that of hPTH' in the treatment of rats osteoporosis. It indicates that hPTH' is better than hPTH (1-34) in osteoporosis treatment once again.

In nasal drug delivery system, different dosage forms could lead to different bioavailability of drugs. Generally speaking, The bioavailability of liquid spray is significantly higher than that of nasal drops. There is no significant difference in the bioavailability between powder spray and liquid spray. However, the chemical stability and microbial stability of powder spray are higher than those of liquid spray. Moreover, because of longer retention time in vivo and 
contact time with nasal mucosa, the bioavailabilities of powder formulations and gel formulations were higher than those of liquid formulations (F. Gu et al. ${ }^{6}$, L. Illum ${ }^{7}$ and S. Lungare et $a l^{8}{ }^{8}$. In this study, nasal drops with the lowest bioavailability in nasal drug delivery system was used in the treatment of rat osteoporosis because of its simplicity and lower risk. Furthermore, the lowest effective dose of hPTH' nasal drops should be effective in other dosage forms on osteoporosis treatment.

\section{Conclusions}

The dosage of $12.5 \mathrm{~g} / \mathrm{rat}$ hPTH' nasal drops could increase the bone mass of OVX rats (especially the cancellous bone mass) and completely cure osteoporosis, while the dosage of $3.125 \mathrm{~g} / \mathrm{rat}$ hPTH' nasal drops could only partially improve osteoporosis. Therefore, the lowest effective dose of hPTH' nasal drops was $12.5 \mu \mathrm{g} / \mathrm{rat}$. hPTH' nasal drops have the potential to be a new therapeutic agent for osteoporosis.

\section{Acknowledgements}

This work was supported by Liaoning Economic Management Cadre Institute (Ljgyktqn1721). Moreover, we thank Kejun You for his help in the experiment in rats.

\section{References}

1. F. Jiao, L. Yanhua, X. Yun, W. Huaqian, L. Taiming, L. Jingjing et al., A novel human parathyroid hormone (1-34) analog for the treatment of osteoporosis, Peptides, 30 (2009) 1173-1180.

2. Marttin E, Verhoef JC, Merkus FW, Efficacy, safety and mechanism of cyclodextrins as absorption enhancers in nasal delivery of peptide and protein drugs, J Drug Target, 6 (1998) 17-36.

3. S. Salatin, J. Barar, M. Barzegar-Jalali, K. Adibkia, M.A. Milani, M. Jelvehgari, Hydrogel nanoparticles and nanocomposites for nasal drug/vaccine delivery, Arch Pharm Res., 39 (2016) 1181-1192.

4. T. Matsumoto, M. Shiraki, H. Hagino, H. Iinuma, T. Nakamura, Daily nasal spray of hPTH(1-34) for 3 months increases bone mass in osteoporotic subjects: a pilot study, Osteoporos Int., 17 (2006) 1532-1538.

5. X. Shuyun, B. Rulian, C. Xiu, Methodology of Pharmacological Experiment, China, 2001, str. 1542.

6. F. Gu, W. Ma, G. Meng, C. Wu, Y. Wang, Preparation and in vivo evaluation of a gel-based nasal delivery system for risperidone, Acta Pharm., 66 (2016) 555-562.

7. L. Illum, Nasal drug delivery--possibilities, problems and solutions, J Control Release, 87 (2003) 187-198.

8. S. Lungare, K. Hallam, R.K. Badhan, Phytochemical-loaded mesoporous silica nanoparticles for nose-to-brain olfactory drug delivery, Int J Pharm., 513 (2016) 280-293. 